\title{
Fungaemia caused by obstructive renal candida bezoars leads to bilateral chorioretinitis: a case report
}

\author{
Johannes Stein ${ }^{1 *} \mathbb{D}$, Stefan Latz', Jörg Ellinger ${ }^{1}$, Guido Fechner ${ }^{1}$, Maher Safi ${ }^{1}$, Philipp Krausewitz ${ }^{1}$, Simone Müller ${ }^{2}$, \\ Karin Weyer ${ }^{1}$ and Stefan C. Müller ${ }^{1}$
}

\begin{abstract}
Background: Renal fungal bezoars are remarkably rare and mostly occur in immunodeficient patients. Only a small number of cases with immunocompetent patients have been published so far. The published treatment approaches comprised systemic antimycotic therapy and surgical or minimal invasive removal of the fungal balls. In some cases irrigation of the renal duct system with amphotericin B was performed.

By obstruction of the urinary tract bezoars can lead to infected hydronephrosis and severe urosepsis with high lethality. Fungaemia can cause fungal colonization in different distant organs. Fulminant chorioretinitis and irreversible visual impairment can be the consequence of ocular fundus colonization. The following report highlights that a co-operation between urologists and ophthalmologists is absolutely indispensible in case of fungaemia.

Case presentation: Hereinafter we describe a case of an immunocompetent 56 years old woman, presenting with flank pain and shivering. The diagnosis turned out to be difficult due to initially negative urine culture. The fungaemia caused by obstructive nephropathy led to bilateral candida chorioretinitis. The patient was treated with intravenous amphotericin b and the bezoar was removed by percutaneous "nephrolitholapaxy".

After two months, a follow up revealed the patient felt well, chorioretinal lesions regressed and urine culture did not show any fungal growth.

Conclusion: To the best of our knowledge, this is the first case reporting on obstructive renal bezoars, which lead to haematogenous fungus spread and bilateral chorioretinitis.

It points out that extensive ophthalmologic examination should be performed in case of fungaemia even if the patient is not suffering from any visual impairment.
\end{abstract}

Keywords: Candida chorioretinitis, Candida glabrata, Fungaemia, Obstructive nephropathy, Renal bezoars

\section{Background}

Renal candida bezoars are remarkably rare and mostly occur in immunodeficient patients. As bezoars can easily be misdiagnosed as urolithiasis a delayed or wrong therapy could be the result.

By obstruction of the urinary tract renal bezoars can quickly lead to fungaemia and cause fulminant disease progression with high lethality.

\footnotetext{
* Correspondence: Johannes.stein@ukb.uni-bonn.de

'Department of Urology, University of Bonn, Sigmund-Freud-Str. 25, D-53127

Bonn, Germany

Full list of author information is available at the end of the article
}

Although an interdisciplinary treatment of a patient by urologists and ophthalmologists is unusual, it is indispensable in case of fungaemia.

This case description outlines particular challenges of the diagnosis of renal bezoars and provides an overview of therapy options.

\section{Case presentation}

In February 2017, a 56-year old woman was transferred from a peripheral hospital to the department of Urology (University of Bonn) due to left sided flank pain and elevated serum infection parameters. An abdominal 


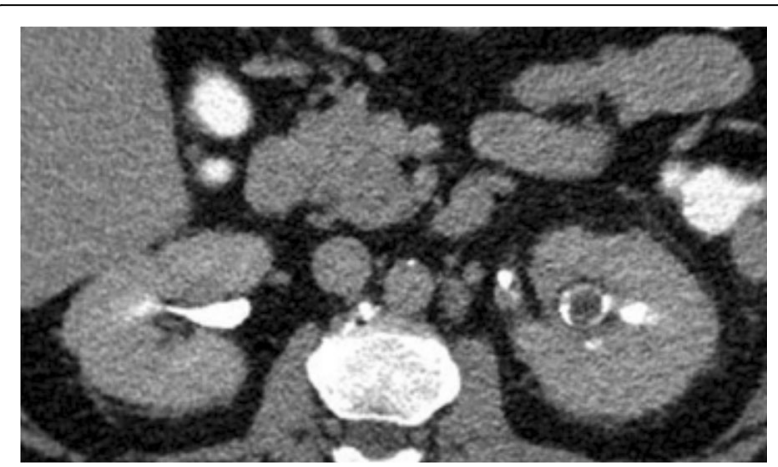

Fig. 1 CT scan showed an obstruction of the renal pelvis caused by an amorphous mass (Hounsfield units: 13)

computed tomography scan showed an obstruction of the renal pelvis caused by an amorphous mass (Fig. 1).

The patient had neither relevant urologic medical history nor hematuria but she reported intermittent shivering since one week. The physical examination showed severe left sided flank pain on palpation. Extensive laboratory examination revealed leucocytosis $(22.53 \mathrm{G} / \mathrm{l})$, elevated C-reactive protein $(253 \mathrm{mg} / \mathrm{dl})$ and creatinin $(1.4 \mathrm{mg} / \mathrm{dl})$ as well as significant leucocyturia. A calculated antibiotic therapy with ceftriaxone and tobramycin was initiated and a double-J-stent was inserted into the left ureter. The urine culture of the admission day did not reveal bacterial growth.

Due to missing clinical recovery with persistent fever and elevated serum infection parameters, the antibiotic treatment was changed to meropenem after four days.
Eventually, repeated urine culture and blood cultures revealed fungaemia with Candida glabrata. Therefore, intravenous antifungal therapy with caspofungin was started. Due to an allergic reaction with exanthema the therapy was shifted to amphotericin B. Blood tests ruled out HIV infection and Diabetes and there was no evidence for other immune deficiencies.

Cerebral and thoracoabdominal computed tomography scan excluded an extrarenal focus of infection and echocardiography did not show any intracardial fungal vegetations. Although the patient did not suffer from any visual impairment we initiated ophthalmoscopic examination as recommended in literature in case of fungaemia [1]. Funduscopy revealed fungal parapapillary chorioretinal infiltrates (Fig. 2). Under the antimycotic treatment, the inflammatory parameters were regressive and the patient's clinical condition improved significantly. Ureterorenoscopic examination revealed a tough yellowish-gray mass in the renal pelvis.

Due to insufficient ureterorenoscopic removal of the mass, it was decided to perform percutaneous "nephrolitholapaxy". Thus the material could be extracted completely (Fig. 3). Microbiological and histological work-up revealed fungal bezoar colonized with Candida glabrata. Seven days after intervention urine culture control proved the absence of fungal colonization.

Finally, after 22 days of amphotericin B therapy, ophthalmologic re-examination showed a complete regression of the chorioretinal candida infiltrates and the patient was discharged in good general condition with normalized infection parameters.

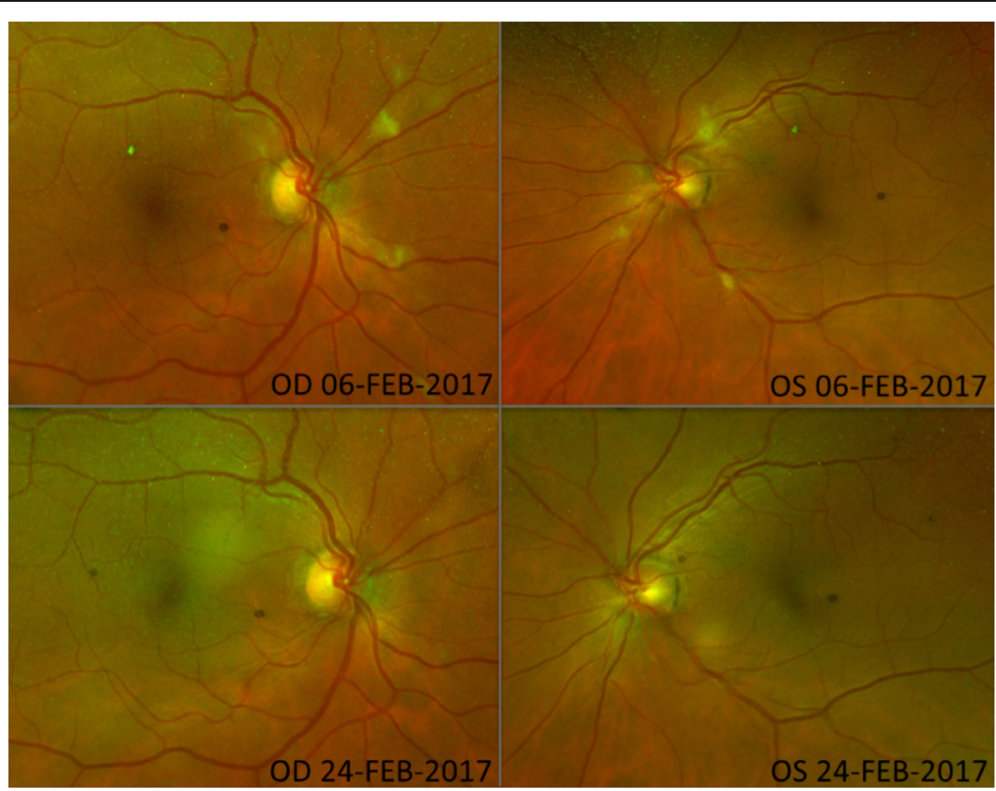

Fig. 2 Funduscopic findings in right eye (OD) and left eye (OS) before (upper line) and after (lower line) initiation of systemic antimycotic treatment. Well demarcated parapapillary retinal infiltrates regress under antimycotic treatment 


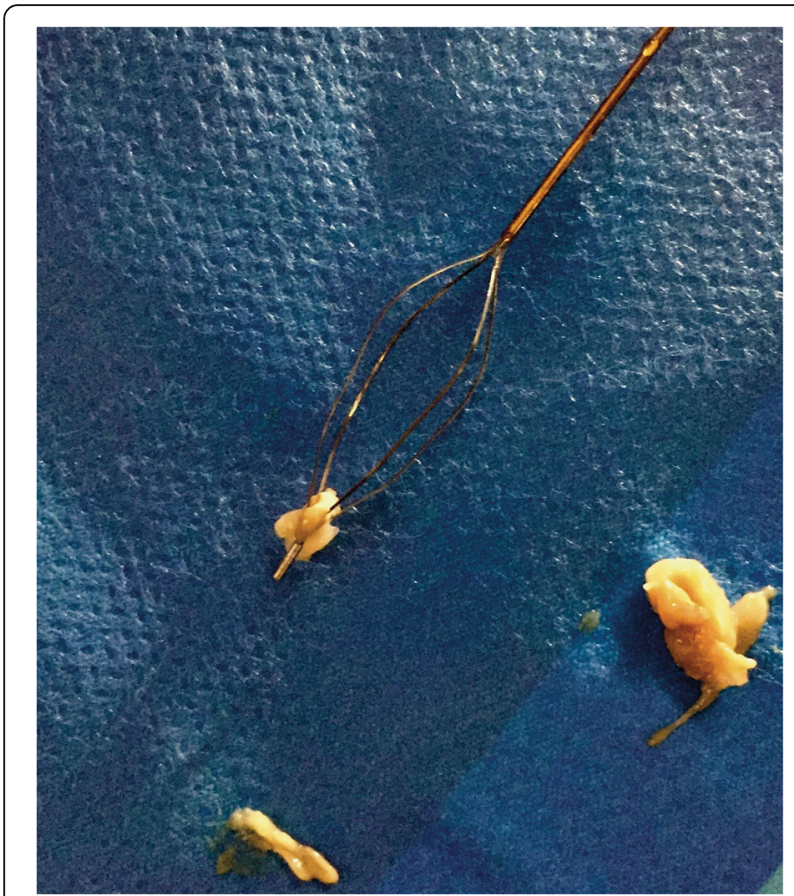

Fig. 3 Parts of the fungal bezoars after removal

After a follow up of two months the patient felt well and urine culture was sterile.

\section{Discussion}

Although yeast infections of the urinary tract are common findings, obstructive uropathy caused by fungal bezoars is exceptionally rare and mostly occur in patients with diabetes or immunosuppression [2-6]. Only a small number of cases with immunocompetent patients have been published so far [7]. These patients were treated with systemic antimycotic agents and by surgical or minimal invasive removal of the fungal balls. In some cases irrigation of the renal duct system with amphotericin B was performed $[2,6,8]$. As our case has shown, it is difficult to remove the viscous material by ureterorenoscopy. It has to be discussed whether an initial percutaneous nephrostomy or an internal DJ stent is preferable in these cases. Arguments for a percutaneous nephrostomy are the direct drainage of the urine and the pre-existing access for the subsequent removal of the bezoars. However, a preinterventional diagnosis is challenging as our case highlights.

\section{Conclusion}

To the best of our knowledge, this is the first case reporting on obstructive renal bezoars, which lead to haematogenous spread and bilateral fungus colonization of the ocular fundus.

It points out that extensive ophthalmologic examination should be performed in case of fungaemia even if the patient is not suffering from any visual impairment.
Antimycotic intravenous therapy should be applied until ocular fungal infiltrates have completely disappeared.

\author{
Acknowledgements \\ Not applicable. \\ Funding \\ There are no funding sources for this case report.
}

Availability of data and materials

All data are included in the manuscript.

Authors' contributions

JS, SL and SM made substantial contributions to conception and design, acquisition of data and have been involved in drafting the manuscript. KW PK and MS made substantial contributions to acquisition of data. SCM, JE and GF revised it critically for important intellectual content. All authors have given final approval of the version to be published. All authors have participated sufficiently in the work to take public responsibility for appropriate portions of the content; and agreed to be accountable for all aspects of the work in ensuring that questions related to the accuracy or integrity of any part of the work are appropriately investigated and resolved.

Ethics approval and consent to participate

Not applicable.

\section{Consent for publication}

We received written consent for publication from the patient.

\section{Competing interests}

The authors declare that they have no competing interests.

\section{Publisher's Note}

Springer Nature remains neutral with regard to jurisdictional claims in published maps and institutional affiliations.

\section{Author details}

'Department of Urology, University of Bonn, Sigmund-Freud-Str. 25, D-53127 Bonn, Germany. ${ }^{2}$ Department of Ophthalmology, University of Bonn, Sigmund-Freud-Str. 25, D-53127 Bonn, Germany.

Received: 18 September 2017 Accepted: 12 March 2018

Published online: 16 March 2018

\section{References}

1. Khalid A, Clough LA, Symons RC, Mahnken JD, Dong L, Eid AJ. Incidence and clinical predictors of ocular candidiasis in patients with Candida fungemia. Interdiscip Perspect Infect Dis. 2014;2014:650235.

2. Jiang SH, Myers RL, Walters GD. Candida tropicalis bezoar as a cause of obstructive nephropathy. Kidney Int. 2011;79(6):690.

3. Arichi N, Yasumoto H, Ogawa K, Nagami T, Anjiki H, Nakamura S, et al. Case report of a ureteral obstruction by Candida Albicans fungus balls detected by magnetic resonance imaging in kidney transplant recipient. Exp Clin Transplant. 2014;12(6):559-61.

4. Irby PB, Stoller ML, MCAninch JW. Fungal bezoars of the upper urinary tract. J Urol. 1990;143(3):447-51.

5. Morris BS, Chudgar PD, Manejwala O. Primary renal candidiasis: fungal mycetomas in the kidney. Australas Radiol. 2002:46(1):57-9.

6. Tan WP, Turba UC, Deane LA. Renal fungus ball: a challenging clinical problem. Urologia. 2016;15:0.

7. Di Paola G, Mogorovich A, Fiorini G, Cuttano MG, Manassero F, Selli C. Candida bezoars with urinary tract obstruction in two women without immunocompromising conditions. ScientificWorldJournal. 2011;11:1168-72.

8. Shimada S, Nakagawa H, Shintaku I, Saito S, Arai Y. Acute renal failure as a result of bilateral ureteral obstruction by Candida albicans fungus balls. Int J Urol. 2006;13(8):1121-2. 